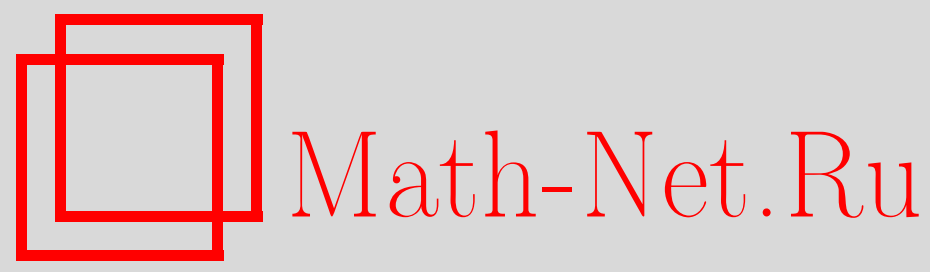

М. Ф. Сухинин, Две теоремы о неподвижной точке, Матем. заметки, 1999, том 66, выпуск 6, 920-923

DOI: https://doi.org/10.4213/mzm1236

Использование Общероссийского математического портала Math-Net.Ru подразумевает, что вы прочитали и согласны с пользовательским соглашением http://www.mathnet.ru/rus/agreement

Параметры загрузки:

IP: 54.157 .27 .8

26 апреля 2023 г., 15:15:24

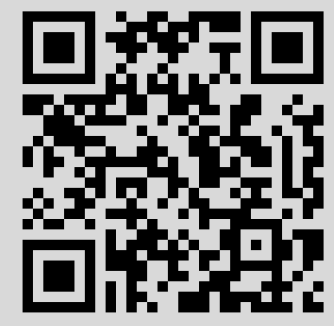


УДК 517

\title{
ДВЕ ТЕОРЕМЫ О НЕПОДВИЖНОЙ ТОЧКЕ
}

\section{М. Ф. Сухинин}

\begin{abstract}
Доказываются две теоремы о неподвижной точке, являющиеся реализациями более общего принципа автора для частично упорядоченных множеств.

Библиография: 3 названия.
\end{abstract}

Напомним результаты из [1] (см. также [2], где приведены более подробные доказательства приложений). Обозначим через $m(X)$ множество максимальных элементов в частично упорядоченном множестве $X$.

1. Теорема [1, с. 42]. Пусть заданы частично упорядоченное множество $M$, $X \subset M$ u $f: X \rightarrow M$. Положим $E=\{x \in X \mid x \leqslant f(x)\}$ u $P=\{x \in E \mid f(x) \leqslant x\}$. Пусть

a) $m(E) \neq \varnothing$;

б) $\forall x \in E \backslash P \quad \exists z \in E:(x \leqslant z) \wedge(z \nless x)$.

Тогда $\exists x \in X: x \leqslant f(x) \leqslant x$.

ДоказАтельство. В силу а) $\exists x \in m(E) \subset E$. Если $x \notin P$, то в силу б) $\exists z \in E$ : $(x \leqslant z) \wedge(z \nless x)$. Тогда $x \notin m(E)$. Следовательно, $x \in P$. Таким образом, $x \leqslant f(x) \leqslant x$.

Несмотря на тривиальность, этот принцип позволил взглянуть с общей точки зрения на принципы Тихонова, Биркхофа-Тарского, автора и некоторые другие (подробнее см. в [1] и [2]), а также выработать новые, два из которых представлены в этой работе.

2. Теорема. Пусть $Q$ - хаусдорфов компакт, $F: Q \rightarrow Q$ - непрерывное отображение, $\rho_{\alpha}: Q \times Q \rightarrow[0,+\infty[, \alpha \in A,-$ некоторые функиии. Для $K \subset Q, K \neq \varnothing$, положим $D_{\alpha}(K)=\sup \left\{\rho_{\alpha}(s, t) \mid s, t \in K\right\}$. Пусть

$$
\begin{gathered}
\forall \alpha \in A \exists q_{\alpha} \in\left[0,1\left[\forall s, t \in Q: \quad \rho_{\alpha}(F(s), F(t)) \leqslant q_{\alpha} \cdot \rho_{\alpha}(s, t),\right.\right. \\
\left(K \subset Q, K \neq \varnothing, \forall \alpha \in A: D_{\alpha}(K)=0\right) \Longrightarrow \operatorname{card}(K)=1
\end{gathered}
$$

(m.е. K состоит из одной точки) и

$$
\forall \alpha \in A: \quad D_{\alpha}(Q)<\infty .
$$

Тогда существует единственная неподвижная точка отображения $F$.

Работа выполнена при финансовой поддержке Российского фонда фундаментальных исследований, грант № 96-01-01130. 
ДокАЗАТЕЛЬСтво. Пусть $M$ - системавсех непустых подмножеств множества $Q \times Q$, упорядоченная по убыванию, $\Sigma$ - система всех непустых компактов в $Q$, а $X=\{S \times S \mid$ $S \in \Sigma\}$. Для $x=S \times S \in X$ положим $f(x)=\{(s, F(s)) \mid s \in S\}$. Покажем, что для соответствующих множеств $E$ и $P$ из п. 1 выполнены условия а) и б). Поскольку $f(Q \times Q) \geqslant Q \times Q$ (так как $F(Q) \subset Q)$, то $E \neq \varnothing$. Пусть $C$ - максимальная цепь в $E$ и $v=\bigcap_{x \in C} x$. Тогда $v=T \times T \in X$ в силу компактности $Q$. Поскольку $f(v) \subset f(x) \subset x$ для каждого $x \in C$, то $f(v) \subset v$, т.е. $f(v) \geqslant v$, и, следовательно, $v \in E$. Далее, поскольку $\forall x \in C: x \leqslant v$ и $C$ максимальна, то $v \in C$ и $v \in m(E)$, т.е. вьполнено а) из п. 1. Пусть $x=S \times S \in E \backslash P$. Тогда $F(S) \subset S$ и $S$ содержит по крайней мере две различные точки. Далее, $F(S) \neq S$, так как если $F(S)=S$, то в силу (2), (3) и (1) $\exists \alpha \in A$ : $0<D_{\alpha}(S)<\infty$ и

$$
\begin{aligned}
\exists q_{\alpha} \in\left[0,1\left[: \quad D_{\alpha}(S)\right.\right. & =\sup \left\{\rho_{\alpha}(F(s), F(t)) \mid s, t \in S\right\} \\
& \leqslant q_{\alpha} \sup \left\{\rho_{\alpha}(s, t) \mid s, t \in S\right\}=q_{\alpha} D_{\alpha}(S),
\end{aligned}
$$

что невозможно. Положим $U=F(S)$ и $z=U \times U$. Тогда $U-$ непустой компакт, $F(U) \subset U$ и, следовательно, $f(z) \geqslant z$, т.е. $z \in E, x \leqslant z$, но $z \nless x$. Таким образом, выполнено условие б) из п. 1. В силу п. $1 \exists x \in X: f(x)=x$, поскольку введенное нами отношение частичного порядка разделяет различные точки, т.е. $\exists S \in \Sigma$ : $S \times S=\{(s, F(s)) \mid s \in S\}$. Пусть $s \in S$. Тогда $\exists t \in S:(s, s)=(t, F(t))$. Это означает, что $t=s$ и $F(t)=s$, т.е. $F(s)=s$. Допустим, что $\exists s_{1} \in Q, s_{1} \neq s: F\left(s_{1}\right)=s_{1}$. Положим $K=\{s\} \cup\left\{s_{1}\right\}$. Тогда в силу (2) $\exists \alpha \in A: \rho_{\alpha}\left(s, s_{1}\right)>0$ и из (1) следует, что

$$
\exists q_{\alpha} \in\left[0,1\left[: \quad \rho_{\alpha}\left(s, s_{1}\right)=\rho_{\alpha}\left(F(s), F\left(s_{1}\right)\right) \leqslant q_{\alpha} \rho_{\alpha}\left(s, s_{1}\right),\right.\right.
$$

что невозможно. Следовательно, $s$ - единственная неподвижная точка отображения $F$.

3. ЗАмЕчАниЕ. На функции $\rho_{\alpha}$ из п. 2 не накладываются требования симметричности, удовлетворения неравенству треугольника, связанности с топологией в $Q$. Тем не менее, связь с принципом сжимающих отображений очевидна.

4. ЗАмечАниЕ. Пространство $Q$ в п. 2 может быть псевдотопологическим компактом (это означает, что каждьй ультрафильтр в $Q$ сходится в псевдотопологии к некоторой точке из $Q$; тогда непрерывность $F: Q \rightarrow Q$ означает, что $F$ переводит сходящиеся базисы фильтров в сходящиеся; подробнее см. в [3]).

5. ТеоремА. Пусть $(\Omega, \Sigma, \mu)$ - пространство с мерой, функиия $\nu: \Omega \times \mathbb{R} \rightarrow \mathbb{R}$ удовлетворяет условию Каратеодори (т.е. $\nu(s, u)$ непрерывна по и на $\mathbb{R}$ почти при каждом $s \in \Omega u \nu(s, u)$ измерима по $s$ nри каждом $u \in \mathbb{R}), p \in] 0,+\infty[u$ оператор $[N(x)](s)=\nu(s, x(s))$ отображсает замкнутый шар $B$ пространства $L_{p}(\Omega)=L_{p}$ в себя. Тогда $N$ имеет неподвижную точку.

ДокАЗАТЕЛЬСТВо. Не уменъшая обшности, можно считать, что $B=\left\{x \in L_{p} \mid\right.$ $\left.\|x\|_{p} \leqslant 1\right\}$. В дальнейшем через $\widetilde{x}$ мы будем обозначать представитель класса $x \in L_{p}$, а если задана функция $\widetilde{x}$, то через $x$ будем обозначать класс, порожденный функцией $\widetilde{x}$. Можно считать, что $N(0) \geqslant 0$. Иначе положим $\Omega^{\prime}=\{s \in \Omega \mid \nu(s, 0) \geqslant 0\}$, $\Omega^{\prime \prime}=\{s \in \Omega \mid \nu(s, 0)<0\}$,

$$
\widehat{\nu}(s, u)=\left\{\begin{array}{cl}
\nu(s, u), & s \in \Omega^{\prime}, \\
-\nu(s,-u), & s \in \Omega^{\prime \prime},
\end{array} \quad[\widehat{N}(x)](s)=\widehat{\nu}(s, x(s))\right.
$$


и заметим, что $\widehat{N}(B) \subset B$ и $\widehat{N}(0) \geqslant 0$. Если мы докажем, что $\exists \widehat{x} \in B: \widehat{N}(\widehat{x})=\widehat{x}$, то для $\widetilde{\widehat{x}} \in \widehat{x}$ положим

$$
\widetilde{x}(s)=\left\{\begin{aligned}
\widetilde{\widehat{x}}(s), & s \in \Omega^{\prime}, \\
-\widetilde{\widehat{x}}(s), & s \in \Omega^{\prime \prime},
\end{aligned}\right.
$$

и заметим, что $x \in B$ и $N(x)=x$. Поэтому будем считать, что $N(0) \geqslant 0$. Положим $M=B, X=\{x \in B \mid 0 \leqslant x \leqslant N(x)\}, f=N$ и заметим, что $0 \in X \neq \varnothing$. Покажем, что вьполняется условие п. 1 . Пусть $C$ - максимальная цепь в $X$. Тогда $C \neq \varnothing$ и $y: x(\cdot) \mapsto \int_{\Omega} x^{p}(s) d \mu(s)$ отображает биективно с сохранением порядка в обе стороны цепь $C$ на цепь $y(C) \subset[0,1]$, которая, очевидно, конфинальна по возрастанию последовательности $a_{n} \in[0,1], a_{n} \leqslant a_{n+1}, \lim _{n \rightarrow \infty} a_{n}=\sup y(C)$. Тем самым, $x_{n}=y^{-1}\left(a_{n}\right)$ конфинальна $C$ по возрастанию, $\widetilde{x}_{n} \nearrow \widetilde{x}_{*}$ почти всюду и согласно теореме Беппо Леви

$$
\int_{\Omega} \widetilde{x}_{*}^{p}(s) d \mu(s)=\sup y(C),
$$

т.е. $x_{*} \in B$. Переходя к пределу в неравенстве $0 \leqslant \widetilde{x}_{n}(s) \leqslant \nu\left(s, \widetilde{x}_{n}(s)\right)$ (почти всюду), получим, что $0 \leqslant x_{*} \leqslant N\left(x_{*}\right)$, т.е. $x_{*} \in X$. В силу условия $(x \in C) \Longrightarrow x \leqslant x_{*}$ и максимальности $C$ получим, что $x_{*} \in C$ и $x_{*} \in m(X)$, т.е. выполнено условие а) в п. 1 . Пусть $x \in X, N(x) \neq x$ и $\widetilde{x} \in x$. Тогда в силу счетной аддитивности $\mu$

$$
\exists \Omega_{1} \subset \Omega, \mu\left(\Omega_{1}\right)>0, \exists \varepsilon>0: \quad\left(s \in \Omega_{1}\right) \Longrightarrow \nu(s, \widetilde{x}(s))>\widetilde{x}(s)+2 \varepsilon .
$$

Положим

$$
\widetilde{x}_{n}(s)= \begin{cases}\widetilde{x}(s)+\frac{\varepsilon}{n}, & s \in \Omega_{1}, \\ \widetilde{x}(s), & s \in \Omega \backslash \Omega_{1}\end{cases}
$$

(можно считать, что $\widetilde{x}(s)$ определена всюду на $\Omega$ ). Тогда $\widetilde{x}_{n}(s) \rightarrow \widetilde{x}(s)$ и $\nu\left(s, \widetilde{x}_{n}(s)\right) \rightarrow$ $\nu(s, \widetilde{x}(s))$ почти всюду. По теореме Егорова

$$
\exists \Omega_{2} \subset \Omega_{1}, \mu\left(\Omega_{2}\right)>0, \exists n_{0} \in \mathbb{N}: \quad\left(n \geqslant n_{0}, s \in \Omega_{2}\right) \Longrightarrow \nu\left(s, \widetilde{x}_{n}(s)\right)>\nu(s, \widetilde{x}(s))-\varepsilon .
$$

Положим

$$
\widetilde{z}(s)= \begin{cases}\widetilde{x}(s)+\frac{\varepsilon}{n_{0}}, & s \in \Omega_{2}, \\ \widetilde{x}(s), & s \in \Omega \backslash \Omega_{2} .\end{cases}
$$

Если $s \in \Omega_{2}$, то в силу (4) и (6) $\widetilde{x}(s)<\widetilde{z}(s) \leqslant \widetilde{x}(s)+\varepsilon<\nu(s, \widetilde{x}(s))-\varepsilon<\nu(s, \widetilde{x}(s))$, а почти при всех $s \in \Omega \backslash \Omega_{2}$ будет $\widetilde{x}(s)=\widetilde{z}(s) \leqslant \nu(s, \widetilde{x}(s))$, т.е. $0 \leqslant x \leqslant z \leqslant N(x)$. Поскольку $x \in B$ и $N(x) \in B$, то $z \in B$. Далее, если $s \in \Omega_{2}$, то в силу (4), (5) и $(6) \widetilde{z}(s) \leqslant \widetilde{x}(s)+\varepsilon<\nu(s, \widetilde{x}(s))-\varepsilon<\nu(s, \widetilde{z}(s))$, а почти при всех $s \in \Omega \backslash \Omega_{2}$ будет $\widetilde{z}(s)=\widetilde{x}(s) \leqslant \nu(s, \widetilde{x}(s))=\nu(s, \widetilde{z}(s))$, т.е. $z \leqslant N(z)$, и, следовательно, $z \in X$, причем в силу (6) $x \leqslant z$ и $z \neq x$. Таким образом, проверено условие б) в п. 1 и, значит, $\exists x \in X$ : $N(x)=x$.

6. ЗАМЕчАНИЕ. При доказательстве п. 5 были использованы следующие средства:

1) сведе́ние к случаю $N(0) \geqslant 0$;

2) аксиома максимальной цепи;

3) “выпрямление" цепи (т.е. биекция на цепь в $[0,1])$ и переход к конфинальной последовательности;

4) теорема Беппо Леви; 
5) счетная аддитивность меры;

6) теорема Егорова;

7) порядковая насьщенность пересечения замкнутого шара в $L_{p}$ с центром в нуле с положительньм конусом.

Приведем один результат, базирующийся на 2)-7) и также доказьваемьй с помощью п. 1.

7. ТеоремА. Пусть $(\Omega, \Sigma, \mu)$ - пространство с б-конечной мерой, $M$ - топологическое векторное пространство классов әквивалентности измеримых и почти всюду конечных функций из $\Omega$ в $\mathbb{R}$ с метризуемой топологией сходимости по мере на каждом множестве из $\Sigma$ конечной меры и естественным отношением порядка, $M_{+}=\{x \in M \mid x \geqslant 0\}, \mathbb{R}_{+}=[0,+\infty[, X$ - ограниченное замкнутое относительно сходимости в $M$ монотонно неубывающ,х последовательностей множество в $M, X \subset M_{+},\left(x, x^{\prime} \in X, x^{\prime \prime} \in M, x \leqslant x^{\prime \prime} \leqslant x^{\prime}\right) \Longrightarrow x^{\prime \prime} \in X$, $\nu: \Omega \times \mathbb{R}_{+} \rightarrow \mathbb{R}$ такова, что $(t \mapsto \nu(t, x(t))) \in M$ при $x \in M_{+}$,

$$
\forall v \geqslant 0: \quad \nu(t, v) \leqslant \varliminf_{u \rightarrow v+0}^{\lim _{\rightarrow}} \nu(t, u), \quad \forall v>0: \quad \varlimsup_{u \rightarrow v-0} \nu(t, u) \leqslant \nu(t, v),
$$

$K: \Omega^{2} \times \mathbb{R}_{+}^{2} \rightarrow \mathbb{R}$ измерима, $K(t, s, u, v)$ монотонно не убивает по и и $v$ (без предположсения о непрерывности справа или слева по и или $v)$, причем определено отобрахсение $f: X \rightarrow M$,

$$
[f(x)](t)=\nu(t, x(t))+\int_{\Omega} K(t, s, x(t), x(s)) d \mu(s),
$$

$f(X) \cap M_{+} \subset X u \exists x_{0} \in X: x_{0} \leqslant f\left(x_{0}\right)$. Тогда $\exists x \in X: f(x)=x$.

8. ЗАМЕЧАНИЕ. При реализации схемы из п. 5 в п. 7 можно использовать для интегральной добавки технику из $[2$, c. 21$]$. В качестве $\nu(t, u)$ в п. 7 может быть взята, например, $\nu(t, u)=\nu_{1}(t, u)+a(t) \nu_{2}(u)$, где $\nu_{1}: \Omega \times \mathbb{R}_{+} \rightarrow \mathbb{R}$ удовлетворяет условию Каратеодори, $a: \Omega \rightarrow \mathbb{R}_{+}$измерима, а $\nu_{2}$ монотонно не убывает (без предположения о непрерывности справа или слева). В качестве $K$ в п. 7 можно взять $K(t, s, u, v)=K_{1}(t, s, u, v)+$ $a_{2}(t, s) K_{2}(u)+a_{3}(t, s) K_{3}(v)$, где $K_{1}: \Omega^{2} \times \mathbb{R}_{+}^{2} \rightarrow \mathbb{R}$ удовлетворяет условию Каратеодори, монотонно не убьвает по $u$ и $v, a_{i}: \Omega^{2} \rightarrow \mathbb{R}_{+}$измеримы, а функции $K_{i}: \mathbb{R}_{+} \rightarrow \mathbb{R}$, $i=2,3$, монотонно не убьвают (без предположения о непрерьвности справа или слева) с соответствующими ограничениями с тем, чтобы удовлетворить условиям $f(X) \cap$ $M_{+} \subset X$ и $\exists x_{0} \in X: x_{0} \leqslant f\left(x_{0}\right)$.

\section{СПИСОК ЦИТИРОВАННОЙ ЛИТЕРАТУРЫ}

[1] Сухинин М.Ф. О трех принципах разрешимости операторных уравнений // Матем. сб. 1993. T. 184. № 1. C. 41-54.

[2] Сухинин М. Ф. Избранные главы нелинейного анализа. М.: Изд. Российского ун-та Дружбы народов, 1992.

[3] Фрелихер А., Бухер В. Дифференциальное исчисление в векторных пространствах без нормы. М.: Мир, 1970. 